\title{
MALIX : une machine 10 GW pour l'émission X intense entre 8 et $10 \mathrm{keV}$
}

\author{
J. Larour, P. Romeas, J. Rous et G. Vu-Tien
}

Laboratoire des Plasmas Denses, CNRS URA 1096, Université Paris 6, boîte 90, 4 place Jussieu, 75252 Paris Cedex 05, France

Intense point-like $X$-ray sources are of interest for plasma diagnostics, especially density measurement at high values in Z-pinches. The device presented here is designed to deliver pulsed power above some GW during 50ns. It is based on a 8 stages Marx generator and a water dielectric pulse forming line. The load is a low inductance vacuum diode with a small emitting zone delivering X-ray bursts on the fundamental lines of the metal material.

La production d'impulsions intenses dans la gamme nanoseconde présente un intérêt certain pour le sondage par projection-absorption (point source X-ray backlighting) [1] de plasmas très denses (Z-pinch à très haute densité, confinement inertiel) ou pour la radiocristallographie éclair de matériaux en évolution rapide (recuit explosif, compression par choc laser) [2]. Un programme de coopération internationale a permis de construire à Paris un

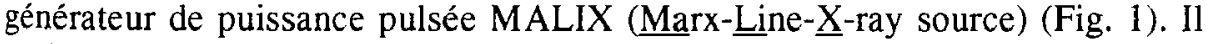
est basé sur la succession de :

-un générateur de Marx compact à 8 étages, isolé par huile ( 8 condensateurs $1,7 \mu \mathrm{F} 30 \mathrm{kV}, 4$ éclateurs à air sec pressurisé) alimenté par un transformateur H.-T. $+V /-V$, et à déclenchement extérieur, -une ligne longue de $2,3 \mathrm{~m}$ constituée de: une ligne à eau de basse impédance pour le stockage de l'énergie et la mise en forme de l'impulsion $\left(\phi_{\text {int }}=168 \mathrm{~mm}\right.$, $\left.\emptyset_{\text {ext }}=219 \mathrm{~mm}, \quad 1=1,7 \mathrm{~m}, \quad \mathrm{Z}=6,7 \quad \mathrm{Ln}\left(\varnothing_{\text {ext }} / \phi_{\text {int }}\right)=1,7 \Omega, \quad \tau=50 \mathrm{~ns}\right)$, un éclateur principal pressurisé au $\mathrm{SF}_{6}$, enfin une section de ligne à eau pour le transfert. 


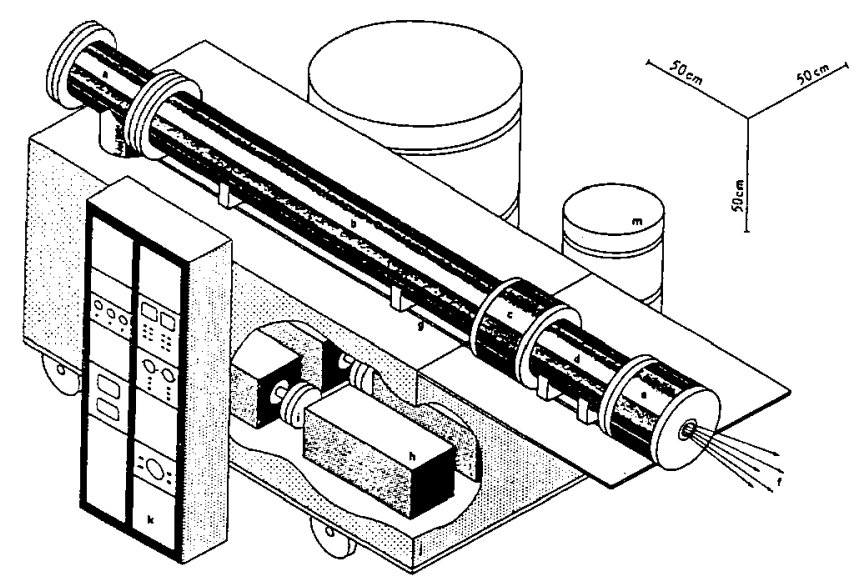

Fig 1. - Vue d'artiste du générateur MALIX a.Té de raccordement sous huile b.Ligne à eau de stockage $\mathrm{l}=1,7 \mathrm{~m} ; \mathrm{Z}=1,7 \mathrm{ohm}$ c.Eclateur principal $\mathrm{SF}_{6}$ d.Ligne à eau de transfert $\mathrm{l}=0,4 \mathrm{~m}$; $\mathrm{Z}=1,7 \mathrm{ohm}$ e.Cellule d'émission f.Faisceau $\mathrm{X}$ g.Rails h.Condensateurs $1,7 \mu \mathrm{F} ; 30 \mathrm{kV}(\mathrm{x} 8)$ i. Eclateur déclenché $(\mathrm{x} 4)$ j.Réservoir blindé k.Panneau de contrôle et commande l.Réservoir d'huile (800l) m.Réservoir d'eau déionisée (601)

[Artist's view of MALIX generator a.oil insulated connection b.storage water line $1=1,7 \mathrm{~m}$; $\mathrm{Z}=1,7 \mathrm{ohm}$ c.SF6 pressurized main switch d.transfer water line $1=0,4 \mathrm{~m} ; \mathrm{Z}=1$,7ohm e.emitting cell f.X ray g.rails h.capacitors $1,7 \mu \mathrm{F} ; 30 \mathrm{kV}(\mathrm{x} 8)$ i.triggered switches $(\mathrm{x} 4)$ j.shielding tank k.control and command rack l.oil storage $(8001)$ m.deionized water storage $(601)]$

Dans les conditions standard $(30 \mathrm{kV})$, la machine stocke 6 kilojoules; le Marx délivre une impulsion de $240 \mathrm{kV}$ en $200 \mathrm{~ns}$, ce qui conduit à une impulsion de courant de $200 \mathrm{kA}$ pendant $100 \mathrm{~ns}$ en bout de ligne. La structure extrêmement repliée de la machine a été choisie pour rendre la source mobile et pour faciliter l'approche d'autres dispositifs, en vue des utilisations mentionnées plus haut. Seule l'utilisation de condensateurs à haute densité d'énergie permettrait de réduire cet encombrement.

Les objets à sonder, plasmas denses créés dans des machines de très forte puissance (térawatt, $10^{12} \mathrm{~W}$ ), sont caractérisés par :

-une petite taille (au maximum millimètre) dans deux directions (colonne) ou dans trois directions (boule) et des forts gradients des paramètres de plasma, -une courte durée de vie (d'une centaine de nanosecondes à une nanoseconde), -une température élevée (plusieurs centaines d'électron-volts, $\mathrm{T}>10^{6} \mathrm{~K}$ ) -une très forte densité (100 à 1000 fois la densité du solide pour des plasmas: d'hydrogène ou de deutérium obtenus à partir de cibles solides cryogéniques).

L'obtention de volumes bien définis à haute densité est un critère de réussite de ces expériences de confinement. La mesure du profil de densité, avec une bonne résolution spatiale, est donc un diagnostic crucial. Comme ces plasmas sont très fortement absorbants pour le rayonnement visible, ultra-violet voire $\mathrm{X}$ mou, il est nécessaire de sonder le plasma avec des outils pénétrant en profondeur. Les plasmas créés par laser ne produisent pas des spectres $X$ suffisamment durs pour pénétrer certains plasmas denses. Avec les rayons $X$ durs (8 à $10 \mathrm{keV}$, longueur d'onde environ $1 \AA ̊$ ) émis en un point de l'anode par 
freinage d'électrons énergétiques, on peut réaliser une projection en absorption du plasma (point source X-ray backlighting). Pour la production de rayons X, notre générateur doit être connecté à une chambre à très faible inductance. Selon les longueurs d'onde souhaitées, on peut utiliser une diode à bombardement d'anode, un plasma type $Z$-pinch à fibre de carbone ou à fils croisés ( $X$-pinch).

Divers montages expérimentaux sont possibles en bande $X$ [1], analogues à ceux de l'optique visible (projection simple sans optique, imagerie par trou d'aiguille). En faisant certaines hypothèses (symétrie géométrique, continuité, nature du milieu absorbant) une analyse numérique de l'image enregistrée dans un plan (film photographique, caméra électronique) permet de reconstituer le profil de densité.

La principale performance demandée au générateur est sa précision de déclenchement et de synchronisation avec le générateur créant le plasma dense. Ceci représente le principal travail de mise au point, actuellement en cours.

[1] Brill, B, Arad, B, Kishenevsky, M, Ludmirsky, A et Zigler, A, J. Phys. D: Applied Phys. 23 (1990) 1069-1072

[2] Wark, J, S, Witlock, R, R, Hauer, A,A, Swain, J,E, et Solone, P,J, Phys. Rev. B35 (1989) 9391-9334, idem B40 (1991) 5705-5714

[3] Favre, M, Proc. of NATO Symp. on Small Scale Laboratory Plasma Experiments, Lee, S, et Sakanak P, H, éd., World Scientific (1989) 170-186

En collaboration : Imperial College, London, G.B. et Pontificia Universidad, Santiago, Chili 